\title{
EVALUACIÓN DE UN MODELO DE CALIDAD DE VIDA CONSTRUIDO DESDE LOS DATOS
}

\author{
Natalia Cancino ${ }^{1}$, Catalina González ${ }^{1}$, Ismael Gallardo ${ }^{1 *}$ y Claudia Estrada ${ }^{2}$ \\ ${ }^{1}$ Universidad de Talca $-{ }^{2}$ Universidad de Magallanes
}

\author{
Recibido, agosto 14/2014 \\ Concepto evaluación, mayo 22/2015 \\ Aceptado, diciembre 8/2015
}

\begin{abstract}
Resumen
La calidad de vida (CV) es un concepto utilizado por diferentes disciplinas. Sin embargo, existen diversas definiciones del mismo, lo que puede llevar a problemas teóricos y aplicados. El objetivo de esta investigación es evaluar un modelo de calidad de vida construido desde los datos. En este trabajo se argumenta que una manera de atender a esta variedad es evaluar un modelo de CV que surja a partir de la aplicación simultánea de instrumentos tradicionalmente vinculados a este constructo. Por ello, se realizaron diferentes análisis factoriales para identificar un factor común a los ítems, y factores específicos en caso de ser necesario. Se presentan los resultados de dos estudios: el primero $(n=550)$ midió diferentes aspectos objetivos y subjetivos relacionados a CV. Los datos generados llevaron a un modelo de seis factores de primer orden y uno de segundo orden, con adecuados indicadores de confiabilidad y validez. Este modelo se basa en variables relativas a los aspectos subjetivos de la CV. El segundo estudio $(n=304)$ revela que la estructura planteada en el primer estudio muestra adecuados indicadores aun comparándola con otros modelos que incluyen dimensiones complementarias. Se discuten los resultados en función de su aporte a la investigación en $\mathrm{CV}$, a la definición conceptual del constructo y a su efecto en la intervención social.
\end{abstract}

Referencia: Cancino, N., González, C., Gallardo, I. \& Estrada, C. (2016). Evaluación de un modelo de calidad de vida construido desde los datos. Acta Colombiana de Psicología, 19(1), 297-309. DOI: 10.14718/ACP.2016.19.1.13

Palabras clave: bienestar, calidad de vida, indicadores sociales.

\section{EVALUATION OF A CONCEPTUAL QUALITY OF LIFE MODEL BUILT FROM DATA}

\begin{abstract}
Quality of Life (QL) is a concept used by different disciplines. However, there is a variety of definitions of QL, which might lead to theoretical and applied problems. The present work argues that a way to attend to this problem is by assessing a model of quality of life that arises from the simultaneous application of instruments traditionally associated with this construct. To evaluate this idea, several factor analyses were conducted in order to identify a common factor and specific dimensions in case it was needed. The first study $(\mathrm{n}=550)$ measured objective and subjective aspects related to QL. The resulting data generated a model with six first order factors and one second order dimension with appropriate indicators of reliability and validity. This model is based on variables related to the subjective aspects of QL. A second study $(n=304)$ indicated that the aforementioned structure shows good psychometric indicators, even in comparison with other models that include complementary dimensions. Results are discussed in terms of their contribution to research in quality of life, the conceptual definition of the construct and its effect on social intervention.

Key words: quality of life, social indicators, well-being.
\end{abstract}

* Facultad de Psicología, Universidad de Talca. Av. Lircay s/n Talca, Chile. Tel. +56-71-2201726 igallardo@utalca.cl Trabajo realizado con aportes de la Dirección de Investigación de la Universidad de Talca. Se enmarca dentro del Acuerdo de Colaboración en Investigación en Psicología Social realizado entre la Universidad de Talca y Universidad de Magallanes, al alero de los Convenios de Desempeño "Bicentenario" celebrados entre el Ministerio de Educación de Chile con la Universidad de Talca y la Universidad de Magallanes, respectivamente. 


\title{
AVALIAÇÃO DE UM MODELO DE QUALIDADE DE VIDA CONSTRUÍDO A PARTIR DOS DADOS
}

\begin{abstract}
Resumo
A qualidade de vida (QV) é um conceito utilizado por diferentes disciplinas. Contudo, existem diversas definições dele, o que pode levar a problemas teóricos e aplicados. O objetivo desta pesquisa é avaliar um modelo de QV construído a partir dos dados. Neste trabalho, argumenta-se que uma maneira de atender a essa variedade é avaliar um modelo de QV que surja da aplicação simultânea de instrumentos tradicionalmente vinculados a esse constructo. Por isso, realizaram-se diferentes análises fatoriais para identificar um fator comum aos itens e fatores específicos, caso seja necessário. Apresentam-se os resultados de dois estudos: o primeiro $(n=550)$ mediu diferentes aspectos objetivos e subjetivos relacionados com a QV. Os dados gerados levaram a um modelo de seis fatores de primeira ordem e um de segunda ordem, com adequados indicadores de confiabilidade e validade. Esse modelo se baseia em variáveis relativas aos aspectos subjetivos da QV. O segundo estudo $(n=304)$ revela que a estrutura proposta no primeiro estudo mostra adequados indicadores ainda a comparando com outros modelos que incluem dimensões complementares. Discutem-se os resultados em função de sua contribuição para a pesquisa em QV, para a definição conceitual do constructo e para seu efeito na intervenção social.

Palavras-chave: bem-estar, qualidade de vida, indicadores sociais.
\end{abstract}

El concepto de Calidad de Vida (CV) ha sido de especial importancia para el desarrollo de la ciencia y la intervención social. Su uso frecuente en diferentes disciplinas como la economía, la sociología, el marketing o la psicología (Juárez \& Contreras, 2012; Sirgy et al., 2006), lo han posicionado como uno de los temas más importantes de las ciencias sociales en el siglo XXI (p.ej., Moyano, 2010). Adicionalmente, este interés científico por la CV es acompañado por un interés desde las políticas públicas nacionales, transformándola en un importante indicador del desarrollo social (Land, Michalos \& Sirgy, 2012).

Una de las limitaciones importantes del concepto de $\mathrm{CV}$ es que existe una gran diversidad de significados para el término según el tipo de disciplina que lo utilice. Por ejemplo, desde la economía, la CV se asocia fuertemente a la idea de que el ingreso nacional de un país, medido por el producto interno bruto (PIB), es la medida más importante para evaluar su bienestar (Moyano, 2010; Ryan \& Deci, 2001). Desde esta perspectiva, la CV se evalúa de acuerdo con la presencia o ausencia de diferentes artículos domésticos en el hogar, de cuya sumatoria se infiere la $\mathrm{CV}$ de quien ha respondido. Ejemplos de estos enfoques en Chile son la encuesta de Caracterización Socioeconómica Nacional (CASEN) o el Índice de Calidad de Vida Urbana (ICVU, Orellana, Bannen, Fuentes, Gilabert \& Pape, 2011).

Desde la psicología, la CV se entiende como bienestar personal, considerando aspectos relativos al logro del placer (p.ej., Diener, 1984) o al crecimiento personal (Ryff, 1989; véase Díaz et al., 2006; Gallardo \& Moyano, 2012; Ryan \& Deci, 2001). De esta forma, la CV sería el resultado de la sumatoria de la satisfacción experimentada en los distintos dominios de la vida de una persona (Felce \& Perry, 1995; Urzúa \& Caqueo - Urízar, 2012) o, dicho de otra forma, el auto-reporte acerca del bienestar personal (Land, et al., 2012). Asimismo, el marketing indica que la CV de los consumidores está determinada por mecanismos de mejora de planes de negocios, precios y distribución de los productos (Sirgy, et al. 2006; Lee \& Sirgy, 2012).

Otra manera de analizar la CV es a partir de sus componentes objetivos o subjetivos. Desde este punto de vista, enfoques como el económico o del marketing se enfocarían en los primeros, mientras la psicología sería un ejemplo de los segundos. Por otra parte, existe un tipo de aproximación que integra los ámbitos objetivos y subjetivos, considerando a la CV como un constructo multidimensional (Ardila, 2003; Cummins \& Cahil, 2000; Moyano \& Ramos, 2007). Existen diferentes ejemplos de este tipo de modelos conceptuales. Uno de ellos es el elaborado por la Organización para la Cooperación y el Desarrollo Económico (OECD, por sus siglas en inglés), el cual considera que la CV debe estar determinada por factores como los ingresos familiares, la calidad de la vivienda, entre otros (OECD, 2015). De estas dimensiones, algunas se definen bajo indicadores estrictamente objetivos (p.ej., la renta neta de las familias) o subjetivos (p.ej., la satisfacción de las familias con su vivienda). Pueden verse otros ejemplos similares en los trabajos de Felce \& Perry (1995) o el modelo de CV Integral, de Verdugo, Gómez \& Arias (2007).

Independientemente de la disciplina profesional o del enfoque en lo objetivo-subjetivo, todos los planteamientos conceptuales sobre la CV utilizan una estrategia hipotéticodeductiva para establecer las variables que la determinan. En función de estas teorías se evalúan los modelos con datos de diferentes muestras. Esta es una estrategia clásica en la generación de conocimiento y su evaluación, pero no está exenta de problemas. Es posible que exista un mode- 
lo teórico bien constituido y aceptado por la comunidad científica, pero que dada esta aceptación, no sea criticado ni cuestionado. Al respecto, Greenwald, Pratkanis, Leippe \& Baumgardner (1986) y Greenwald \& Pratkanis (1988) proponen que este fenómeno se debe a una forma de procesar la información consistente con las ideas planteadas por la teoría, lo que reduce, por tanto, la probabilidad de que sea discutida.

En el contexto del estudio de la CV (entendida desde un punto de vista psicológico), existen modelos bien constituidos teóricamente y que se utilizan sistemáticamente en la investigación y en la intervención social. Esta consistencia ha llevado a la generación de explicaciones sobre cómo las personas se orientan al bienestar (p.ej., Ryff, 1989) y a la generación de indicadores nacionales relevantes para la política pública que se basan en estas conceptualizaciones (p.ej., Encuesta Nacional de Desarrollo de la Mediana Edad de Estados Unidos, MIDUS en su sigla en inglés (MIDUS, 2015). No obstante, algunas investigaciones han mostrado que la estructura teórica planteada para entender el bienestar psicológico no es la más adecuada para dar cuenta de la información recogida. Incluso, se ha encontrado que modelos teóricos "absurdos" muestran mejores indicadores de ajuste teórico que el original (Springer \& Hauser, 2006; Springer, Hauser \& Freese, 2006).

Para superar este problema, Greenwald, et al. (1986) han sugerido complementar un acercamiento basado puramente en la teoría, con uno basado en los resultados. Es decir, evaluar la existencia de fenómenos y teorías en función de los datos recogidos, dejando de lado potenciales teorías creadas a priori. De este modo, el sesgo de confirmación que podría estar en la base del uso continuado de ciertas teorías se reduce, dada la inexistencia de un modelo conceptual a priori que se busque investigar.

Dados los problemas conceptuales existentes en el ámbito de la CV y las potenciales ventajas de un enfoque basado en los resultados, este trabajo busca evaluar la existencia de regularidades en diferentes medidas usadas para medir $\mathrm{CV}$. Así, se espera obtener una estructura teórica de CV teniendo como base un enfoque basado en los resultados. De este modo, se desarrolla una propuesta conceptual con base en los datos obtenidos.

Para obtener un modelo de CV desde los datos, se realizará una aproximación que implica la recogida de una gran cantidad de información sobre variables que tradicionalmente se han relacionado con CV. Por ejemplo, el ingreso económico se considera una variable de gran relevancia para la evaluación de la CV en el ámbito económico (Sirgy, et al. 2006). El bienestar psicológico y subjetivo son considerados como formas de comprender la CV desde la psicología, teniendo en cuenta sobre todo aspectos subjetivos como la satisfacción con la vida y el crecimiento personal (Ryff, 1989; Ryff \& Keyes, 1995; Ryff \& Singer, 1998). De la misma forma, el optimismo disposicional ha estado fuertemente relacionado con la CV desde el punto de vista psicológico (p.ej., Concha et al., 2012), evidenciándose que a mayor optimismo, mayor bienestar.

Por otra parte, las personas que contemplan las consecuencias futuras de sus acciones, tienden a pensar en salidas alternativas a situaciones que les producen efectos emocionales negativos, reduciendo así su impacto en el bienestar (Strathman, Gleicher, Boninger \& Edwards, 1994). Aun cuando la CV, desde las diferentes disciplinas, puede no incluir estas variables en su estructura conceptual, lo cierto es que la diversidad de definiciones permite pensar que es posible que existan mejores factores que los actuales para describirla. En un intento por añadir variabilidad a la potencial estructura de datos resultante, se incluyen estas últimas variables.

Una vez recogida esta información se procederá a generar la estructura teórica con el apoyo de procedimientos estadísticos. Después de obtenerla se comparará con otras que puedan ser potenciales explicaciones alternativas o con modelos conceptuales ya existentes. Con base en lo anterior, de este análisis se podrían esperar tres posibles resultados: Primero, que aquellos elementos relacionados con el componente objetivo de la $\mathrm{CV}$ se agrupen en un factor. En segundo lugar, que solo los elementos subjetivos compartan algo en común y que, en su conjunto, conformen la CV. Por último, podría ocurrir que tanto elementos objetivos como subjetivos converjan en un factor común, convirtiéndose en una variable latente que se denominaría CV.

\section{MÉTODO GENERAL}

\section{Descripción general}

Dos estudios transversales de alcance correlacional fueron realizados para cumplir con el objetivo de investigación. En el primero de ellos se recogieron datos de diferentes medidas de calidad de vida en su dimensión subjetiva y objetiva, así como otras adicionales que pudieran ser de relevancia dada su relación con las dimensiones antes mencionadas. Posteriormente, se realizaron diversos análisis estadísticos para extraer, de la información recogida, una estructura de factores que se ajuste de mejor manera a los datos. Esta misma estructura se comparará con otros modelos teóricos que puedan también dar cuenta de la información recogida. El modelo resultante podrá llamarse, para efectos de esta investigación, modelo de calidad de vida. En el segundo estudio se evalúa esta estructura resultante en una muestra 
diferente a la original, y se compara con otras estructuras conceptuales cuyas nuevas dimensiones puedan entregar información adicional al modelo propuesto. No obstante, si la estructura evaluada es estable, esta no debería verse modificada en función de la muestra o de las nuevas dimensiones incluidas. Es importante mencionar que, dada la cantidad de variables y factores a evaluar, se ha optado por utilizar versiones breves de la mayoría de los instrumentos utilizados.

\section{ESTUDIO 1}

En el estudio 1 se busca formular un modelo de calidad de vida a partir de una serie de medidas de la misma, tanto objetivas como subjetivas, y de variables que han sido relacionadas con dicho constructo.

\section{MÉTODO}

\section{Participantes}

Se utilizó una muestra no probabilística compuesta por 550 personas, trabajadoras, quienes participaron voluntariamente en la investigación. Un $66 \%$ eran mujeres y un $34 \%$ eran hombres (28\% de la muestra no respondió a esta pregunta) con edades que fluctuaban entre los 17 y 80 años $(M=39 ; S D=12,5)$. Los participantes provenían en un 99\% de la VII Región del Maule, en Chile.

\section{Instrumentos}

Bienestar Psicológico (BP): Es una de las dimensiones de calidad de vida desde el punto de vista psicológico. Se mide a través de la escala de BP de Ryff (1989; Ryff $\&$ Keyes, 1995), la cual se compone de seis factores: autonomía (AUTO), relaciones sociales positivas (RSP), dominio del entorno (DE), crecimiento personal (CP), auto aceptación (A) y propósito en la vida (PV). Se utilizó una versión abreviada de dicho instrumento, creada a partir de los datos obtenidos en investigaciones previas (Gallardo \& Moyano, 2012). Siguiendo los criterios de trabajos anteriores (Ryff \& Keyes, 1995) se creó una versión de 18 ítems organizados en una escala tipo Likert que va de 1 (Totalmente en desacuerdo) a 6 (Totalmente de acuerdo), a partir de aquellos que poseían una mayor correlación ítem-total. Estudios previos muestran que la escala creada en función de esta estrategia metodológica cuenta con adecuados indicadores psicométricos (p.ej., Ryff \& Keyes, 1995). Para este estudio la confiabilidad total de la escala (por alfa de Cronbach) fue de .91.

Bienestar Subjetivo (BS): Es otra de las dimensiones de calidad de vida desde el punto de vista psicológico. Diener (1994) planteó un modelo del BS compuesto por tres dimensiones: Satisfacción con la Vida (SV), Afecto Positivo (AF) y Afecto Negativo (AN). Para la primera dimensión se utilizó la escala de SV (Diener, 1994), en formato Likert, con rangos de 1 (Totalmente en desacuerdo) a 6 (Totalmente de acuerdo). Para el caso de las dimensiones de afecto positivo y negativo, se ha utilizado un ítem de felicidad, considerando que algunos autores han sugerido que la felicidad está constituida por la sustracción entre afecto positivo y negativo (p.ej., Diener, 1994). Este ítem de felicidad global ha sido utilizado en diferentes investigaciones, mostrando altas correlaciones con diferentes escalas de bienestar subjetivo y felicidad (Bradburn, 1969; Lyubomirski \& Lepper, 1999). En concreto, "en general, considero que soy una persona feliz", en una escala de 1 a 7 (totalmente en desacuerdo-totalmente de acuerdo). El modelo de BS es considerado por diversos estudios como un modelo estable en cuanto a su validez de constructo, evidenciando además una buena confiabilidad general (Diener, Emmons, Larsen \& Griffin, 1985; Moyano \& Ramos, 2007).

Indicadores Económicos: Es una de las maneras de evaluar la calidad de vida desde un punto de vista económico y objetivo. Los indicadores económicos fueron recogidos mediante ítems, en los cuales la persona responde en una escala de quintiles su ingreso mensual aproximado. Esta forma de recoger la información de ingresos económicos se utiliza para conocer la distribución del ingreso en el análisis de la encuesta CASEN (Gobierno de Chile, 2009). Un segundo indicador se forma a partir de la selección que la persona realiza de una lista de diez enseres que pueda tener en su hogar (p.ej., microondas, refrigerador), también usado en la misma encuesta. Una mayor cantidad de enseres seleccionados sugiere mayor ingreso del grupo familiar

GHQ-12: Se utilizó la escala General Health Questionnaire (GHQ-12, por sus siglas en inglés), elaborada por Goldberg y Williams en 1988, validada en población española (Rocha, Pérez, Rodríguez-Sanz, Borrell \& Obiols, 2011). Es un instrumento que detecta la morbilidad psicológica y posibles casos de trastornos psiquiátricos y que ha sido utilizada como una medida de calidad de vida en el ámbito de la salud (p.ej., Malley et al., 2012; Montaezri et al., 2003). Está compuesto por doce ítems, medidos en una escala que va de 0 (mucho menos de lo habitual) a 3 (mucho más de lo habitual) y presenta una confiabilidad con valores entre .82 y .86 (Rocha, et al., 2011).

Trabajo y Familia (TF): Se utilizó una versión reducida de la escala SWING sobre conflicto TF, validada en población española y adaptada a población chilena (Riquelme, Rojas \& Jiménez, 2012), que utiliza una medida de 0 (Nunca) a 
3 (Siempre). Estos estudios muestran que la escala posee indicadores psicométricos adecuados, describiéndose una confiabilidad de entre .77 y .89 y una validez de constructo que muestra la estructura teorizada de cuatro factores.

Optimismo: Se utilizó la versión revisada del Life orientation test (LOT - R. por sus siglas en inglés), compuesto por seis ítems. Esta escala evalúa diferencias individuales entre optimismo y pesimismo, va de 0 a 4 , rango dentro del cual la persona debe evaluar afirmaciones considerando su grado de acuerdo o desacuerdo con ellas. Cuenta con indicadores de confiabilidad (alpha de Cronbach) superiores a .75 (Scheier, Carver \& Bridges, 1994).

Consideración de las consecuencias futuras: Esta variable fue medida a través de la escala de consideración de consecuencias futuras (Strathman, et al., 1994), adaptada para este estudio. Permite establecer diferencias individuales entre las consideraciones que las personas hacen sobre las consecuencias futuras que tendrán sus comportamientos actuales y la medida en que dichos comportamientos se ven influenciados por estos resultados potenciales. La escala se compone de doce ítems, medidos de 1 (Para nada característico) a 7 (Totalmente característico). Esta escala presenta una sola dimensión teórica (Petrocelli, 2003) y su fiabilidad (alpha de Cronbach) para el presente estudio fue de .71

Procedimiento

1. Un grupo de encuestadores entrenados presentó a los participantes un instrumento de autoaplicación compuesto por las escalas descritas previamente.

2. Todas las personas leyeron un consentimiento informado en donde se explicaba el objetivo general de la investigación y los aspectos éticos de la misma.

3. Una vez firmado el documento, los participantes leyeron las instrucciones en compañía de los encuestadores, quienes resolvían las dudas que surgían al responder.

4. Una vez terminada la aplicación, se agradeció a cada uno de los participantes por su colaboración.

\section{Plan de Análisis}

Debido a que las métricas de cada escala aplicada variaban entre sí (lo que podría afectar a los pesos factoriales), se optó por estandarizar las puntuaciones de la totalidad de los participantes, las que fueron utilizadas para los análisis posteriores. En segundo lugar, se realizaron análisis factoriales para establecer las dimensiones que resumían la información recogida. Para ello, se seleccionaron dos sub muestras aleatorias de la muestra total para reducir así la capitalización del azar. Este fenómeno tiende a sesgar hacia los extremos superiores las estimaciones y estadísticos (Cudeck \& Brown, 1983). Por esta razón, no se recomienda realizar análisis factoriales exploratorios y confirmatorios utilizando los mismos participantes. Por lo tanto, a modo de evitar este problema, se utiliza una segunda muestra independiente para probar el modelo (Batista - Fouget, Cohenders \& Alonso, 2004; Carrasco, Delgado, Barbero, Holgado \& del Barrio, 2011; Leiva \& Gallardo, 2013).

Una vez establecidas las muestras, se siguieron diferentes pasos para la creación del modelo conceptual. Primero, se estableció un factor común que reunía aquellos ítems que compartían varianza. Para ello, se realizó un análisis factorial exploratorio sobre una de las muestras seleccionadas, estableciéndose como resultado a priori del mismo la obtención de dos dimensiones. Los ítems que se agruparon en el primer factor fueron seleccionados, asumiendo que resumen lo que comparten del constructo $\mathrm{CV}$.

El procedimiento de reducción de datos fue de máxima verosimilitud, con extracción varimax. Previamente se evaluó la factibilidad de agrupar los datos en un análisis factorial a través de medidas de adecuación muestral. Tanto los resultados de la prueba $\operatorname{KMO}(0,829)$ como Bartlett $(16,849 ; p<.01)$ mostraron indicadores que permiten la realización de dicho análisis. En cuarto lugar, se realizó un Análisis Factorial Exploratorio en la segunda sub muestra, para analizar la estructura del factor obtenido, usando los mismos procedimientos ya descritos. Esto se llevó a cabo para evaluar la posibilidad de que el factor encontrado pudiera dividirse en otros factores más específicos.

Finalmente, para evaluar el grado de ajuste a los datos del modelo encontrado (i.e., su validez de constructo, Ruiz, Pardo \& San Martín, 2010), se realizaron diferentes análisis de ecuaciones estructurales utilizando el programa AMOS 16.0. De acuerdo con lo planteado por Hu \& Bentler (1999) y por Schreiber, Stage, King, Nora \& Barlow (2006), para valorar la adecuación de los modelos se debe utilizar la información aportada por un conjunto de indicadores, absolutos y relativos, con cuyo análisis simultáneo se debe deducir la bondad de ajuste del modelo estudiado.

El análisis utilizó el procedimiento de máxima verosimilitud y, siguiendo las recomendaciones de la literatura, se utilizaron los siguientes indicadores: Chi cuadrado (valores mayores a .05 indican un mejor ajuste del modelo), AIC (Akaike Information Criterion, donde valores más bajos indican un mejor ajuste), RMSEA (Root Mean square error of aproximation, valores menores a .05 son óptimos, aquellos entre .06 a .08 se consideran aceptables) y CFI (Comparative fix index, en que valores mayores a $.95 \mathrm{se}$ consideran aceptables; véase Díaz, et al. 2006; Gallardo \& Moyano, 2012). 


\section{RESULTADOS}

A continuación se presentan los estadísticos descriptivos de las dimensiones involucradas en el estudio, los procedimientos de análisis factorial y la evaluación del ajuste del modelo obtenido de los análisis previos mediante ecuaciones estructurales.
La tabla 1 muestra las medias y la desviación típica de las escalas utilizadas en el primer estudio. A diferencia del resto de variables en general, se observa un sesgo en las respuestas sobre bienestar y felicidad, sugiriendo que las personas poseen una percepción positiva acerca de sí mismas en estas dimensiones.

Tabla 1.

Estadísticos descriptivos de las escalas utilizadas en el estudio 1

\begin{tabular}{ccc}
\hline & M & SD \\
\hline Bienestar Psicológico & $\mathbf{4 , 1}$ & $\mathbf{0 , 9 6}$ \\
\hline Relaciones positivas & 3,8 & 1,18 \\
Autonomía & 3,8 & 1,3 \\
Dominio del entorno & 3,7 & 0,93 \\
Propósito en la vida & 4,2 & 1,5 \\
Autoaceptación & 4,1 & 1,3 \\
Crecimiento personal & 4,5 & 1,4 \\
\hline
\end{tabular}

\begin{tabular}{ccc}
\hline SWING & & \\
\hline Equilibrio negativo Trabajo y Familia & .95 & .6 \\
Equilibrio negativo Familia - Trabajo & .62 & .68 \\
Equilibrio positivo Trabajo Familia & 1,6 & .68 \\
Equilibrio positivo Familia - Trabajo & 1,9 & .73 \\
\hline Optimismo & $\mathbf{2 , 2}$ &. $\mathbf{4 7}$ \\
\hline CFC & $\mathbf{4}$ & .7 \\
\hline GHQ - 12 & $\mathbf{1 , 7}$ &. $\mathbf{3 7}$ \\
\hline Felicidad & $\mathbf{5 , 7}$ & $\mathbf{1 , 1}$ \\
\hline
\end{tabular}

Utilizando la primera de las sub muestras del estudio, se realizó un Análisis Factorial Exploratorio con todos los ítems de la batería aplicada, limitando la obtención máxima de factores posibles a dos. Para maximizar la varianza explicada con la menor cantidad de ítems posible, se seleccionaron aquellos cuyas cargas factoriales fueron iguales o superiores a 0.50 . De este modo se podría tener una escala breve para evaluar el constructo. El primer factor arrojado explica un $14,4 \%$ de la varianza total e incluye 21 ítems. El segundo factor representa un $10,6 \%$ de la varianza explicada e incluye once ítems, todos relativos al equilibrio familia-trabajo. En este sentido, se entiende que el primer factor representa un fenómeno diferente al segundo de ellos.

Después de identificar que dentro del primer factor obtenido sus ítems representaban diferentes dimensiones teóricas (descritas en sus propios instrumentos), se evaluó esta posible multidimensionalidad mediante un Análisis Factorial Exploratorio (realizado en la segunda sub muestra). De este análisis se obtuvieron tres factores, cuyos ítems pertenecen a las escalas de BS (cinco ítems), BP(doce ítems) y, TF (cuatro ítems) (véase apartado instrumentos). En su conjunto explican un $55 \%$ de la varianza total del factor común. 
Para evitar la capitalización del azar, este procedimiento se realizó de manera cruzada en dos muestras seleccionadas de la muestra total de manera aleatoria. En ambos casos los ítems seleccionados por el análisis fueron los mismos. De manera interesante, este muestra que los ingresos y enseres (i.e., la dimensión objetiva de la $\mathrm{CV}$ ) no forman parte del primer factor.

Evaluación del Modelo mediante Ecuaciones Estructurales

Para evaluar el ajuste del Modelo de CV encontrado, los datos del mismo fueron comparados con cinco combinaciones de las dimensiones arrojadas por el procedimiento de análisis factorial. El primer modelo propone la $\mathrm{CV}$ como una variable latente, compuesta por SV, BP, TF (véase apartado instrumentos), y la sumatoria de ingresos y enseres $(\mathrm{IE})^{1}$. El segundo, propone la CV como una variable latente, cuyos componentes son SV y las seis dimensiones del BP: PV, A, CP, Auto y DE; TF y la sumatoria de IE. El tercer modelo considera las mismas dimensiones del modelo 2, pero sin el componente económico. El cuarto, propone la $\mathrm{CV}$ como una variable latente, cuyos factores son, separadamente, la SV, cuatro dimensiones del BP (PV, A, CP y DE), TF y la suma del reporte de ingresos y enseres. Por último, el modelo 5 (véase figura 5), encontrado en el AFE ya descrito, considera a la CV como una variable latente, que se compone de SV, cuatro dimensiones del BP (PV, A, CP Y DE) y TF.

Tabla 2.

Indicadores de ajuste de modelos de $\mathrm{CV}$ analizados

\begin{tabular}{lcccc}
\hline Modelo & Chi cuadrado & AIC & RMSEA & CFI \\
\hline Modelo 1 & 19.3 & 43.304 & .118 & .965 \\
Modelo 2 & 56.467 & 104.467 & .054 & .983 \\
Modelo 3 & 34.371 & 76.371 & .049 & .990 \\
Modelo 4 & 48.47 & 90.478 & .063 & .982 \\
Modelo 5 & 26.74 & 62.74 & .056 & .991 \\
\hline
\end{tabular}

Como se puede observar en la tabla 2, el modelo 5 es el que presenta los mejores indicadores de ajuste, analizando en su conjunto tanto índices absolutos como relativos.

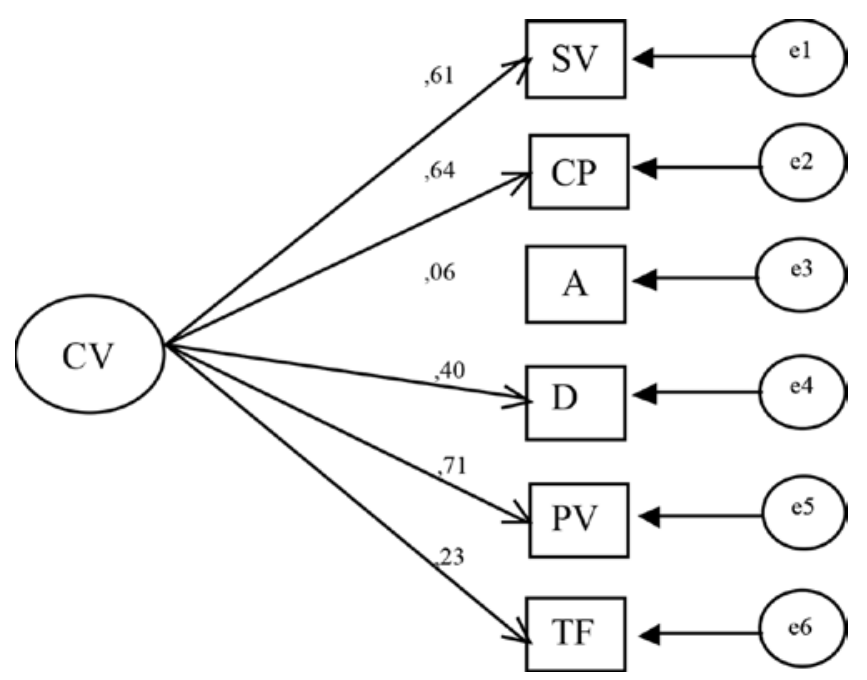

Figura 1. Modelo de Calidad de Vida con seis factores observados

1 Debido a la baja carga factorial arrojada por el autorreporte de ingresos y de enseres se optó por realizar los siguientes análisis con la suma de ambas variables, considerando que, en su conjunto, son un reflejo de la dimensión del llamado componente económico de la CV. 


\section{Análisis de confiabilidad}

Para evaluar la consistencia interna del modelo de seis factores, se utilizó el método de alfa de Cronbach, complementado por el método de dos mitades, debido a que la escala posee más de 20 ítems (pudiendo verse afectada la confiabilidad por dicha cantidad), y a que el modelo factorial arrojó una composición multidimensional del constructo (Oviedo \& Campo - Arias, 2005).

Tabla 3.

Análisis de la consistencia interna de la Escala de CV

\begin{tabular}{|c|c|c|c|c|c|c|}
\hline \multirow{4}{*}{ Alfa de Cronbach } & Dimensión & $\alpha$ & Dimensión & $\alpha$ & $\begin{array}{l}\text { Método } \\
\text { División por mitades }\end{array}$ & \\
\hline & $\mathrm{DE}$ & .49 & A & .73 & Correlación entre las partes & .82 \\
\hline & $\mathrm{CP}$ & .81 & SV & .89 & & \\
\hline & PV & .80 & $\mathrm{TF}$ & .81 & Correccion spearman - Brown & .90 \\
\hline
\end{tabular}

*Nota: $\alpha$ (Valor coeficiente) DE (Dominio del entorno); CP (Crecimiento Personal); PV (Propósito en la vida); A (Auto aceptación); SV (Satisfacción con la vida); TF (Equilibrio Trabajo y Familia)

La tabla 3 muestra que la escala posee buenos indicadores de confiabilidad total y por dimensión, excepto el factor Dominio del Entorno, el cual arroja un bajo coeficiente de consistencia interna. No obstante, este resultado ya se ha observado en investigaciones previas en donde se utiliza el mismo método de reducción de ítems que los aquí utilizados (Ryff \& Keyes, 1995).

\section{DISCUSIÓN}

Los resultados de este primer estudio muestran que los aspectos subjetivos de la CV son los que mejor cuenta pueden dar de los datos recogidos. El modelo obtenido se ajusta mejor a los datos que cualquier otro modelo de los evaluados, incluyendo aquellos que incorporan los factores objetivos. En consecuencia, se llamará a la estructura teórica obtenida, Modelo de Calidad de Vida.

Estos primeros resultados son relevantes a la hora de considerar el aporte para la CV de aspectos subjetivos y objetivos. La estructura obtenida es consistente con la idea de CV que posee la psicología y con modelos psicológicos que incluyen los aspectos tanto hedónicos como eudaimónicos (Ryan \& Deci, 2001). También es consistente con el aspecto central de la definición de la OMS, referida a la percepción que las personas poseen de diferentes aspectos de su vida. De manera importante y novedosa, el componente familiar y del trabajo aparece como factor dentro de este modelo, algo no encontrado en trabajos previos.

No obstante estos resultados, existen algunas limitaciones. Por ejemplo, la muestra utilizada es de trabajadores, lo que lleva a preguntarse si el modelo y sus dimensiones están circunscritos a este tipo de población (por ejemplo, la dimensión laboral y de familia). Por otro lado, la zona territorial en que esta información fue recogida posee características físicas y ambientales que no necesariamente se dan en otras zonas del país o del mundo como, por ejemplo, las regiones sensibles a movimientos telúricos. Ello podría afectar la conformación de la estructura general del modelo propuesto; por ejemplo, la muestra podría ser más sensible a aspectos subjetivos (y menos a aspectos objetivos) debido a la necesidad de afrontar constantemente problemas ambientales graves. Por estas dos razones, se realizó un segundo estudio con una muestra heterogénea de habitantes de la ciudad de Punta Arenas, Chile, distante 2750 kilómetros al sur de la región del Maule. Si el tipo de personas que responde o el tipo de ambiente son responsables de los resultados surgidos en este primer estudio, la estructura factorial propuesta debería mostrar peores indicadores de ajuste que otras alternativas conceptuales.

En segundo lugar, es posible que los aspectos económicos sean importantes para las personas pero no en su dimensión objetiva, sino en la subjetiva. Es decir, la percepción de la propia realidad económica en vez de la situación misma. Un antecedente de esta idea es el aportado por Wolbring, Keuschningg \& Negele (2011), quienes encontraron que el ingreso relativo tiene una mayor relación con la satisfacción con la vida (un indicador de CV psicológica) que el ingreso absoluto. De ser así, el modelo propuesto en el estudio 1 deberá mostrar un mejor ajuste a los datos que el del estudio 2 cuando se introduzca una dimensión re- 
lativa a la percepción subjetiva de la situación financiera/ económica de la persona.

\section{ESTUDIO 2}

En función de los antecedentes anteriores, el segundo estudio buscó evaluar, por un lado, la estructura teórica encontrada en el estudio 1 utilizando una muestra diferente de personas. Por otro, buscó evaluar su ajuste en comparación con los mismos modelos del estudio 1 , y otro adicional que incluye la percepción subjetiva del impacto de los ingresos económicos sobre la vida cotidiana. El procedimiento seguido fue idéntico al descrito en el estudio 1.

\section{MÉTODO}

En el estudio 2, se evaluó el ajuste del modelo obtenido a partir de los resultados del estudio 1 mediante ecuaciones estructurales y se comparó con otro modelo al cual se le incorporó un componente subjetivo de los ingresos económicos.

\section{Participantes}

Se utilizó una muestra no probabilística, intencionada, compuesta por 304 personas, de la ciudad de Punta Arenas, Chile, quienes participaron de forma voluntaria en este estudio. Un $41 \%$ eran hombres y un 59\% eran mujeres, cuyas edades fluctuaban entre los 17 y 79 años $(M=34$; $S D=14,5)$. El procedimiento y el plan de análisis fueron los mismos referidos en el estudio 1 .

Instrumento

Con base en la estructura definida en el estudio 1, se creó una escala tipo Likert de 21 ítems cuyos extremos corresponden a "Totalmente en desacuerdo" (1) y a "Totalmente de acuerdo" (7).

El instrumento contiene seis dimensiones: $\mathrm{CP}$ (crecimiento personal, tres ítems), A (auto aceptación, tres ítems), DE (dominio del entorno, dos ítems), PV (propósito en la vida, tres ítems), SV (satisfacción vital, cinco ítems), TF (trabajo y familia, cuatro ítems), A (autonomía), reporte de ingresos del hogar y posesión de enseres (véase apartado instrumentos). Adicionalmente, se agregó un ítem de la dimensión de Autonomía del BP de modo que agregara mayor variabilidad a esta medida("Tengo confianza en mis opiniones incluso si son contrarias al consenso general"). Este ajuste se realizó debido a la similitud de los indicadores de bondad de ajuste arrojados para los modelos 3 y 4 en el estudio 1 (véase tabla 1). Junto a ello, se incluyeron dos ítems adicionales, correspondientes a la percepción subjetiva del impacto de los ingresos económicos. Concretamente, estos fueron: "Tengo los recursos económicos que necesito para satisfacer mis necesidades" y "Me siento conforme con lo que poseo materialmente".

\section{Procedimiento}

Se utilizó el mismo procedimiento del estudio 1.

\section{RESULTADOS}

Para evaluar el ajuste del Modelo de CV, junto con el ajuste de otros modelos propuestos empíricamente, se comparó la escala de $\mathrm{CV}$ con cinco modelos alternativos usando el mismo procedimiento que en el estudio 1. El primer modelo factorial propone la $\mathrm{CV}$ como una variable latente, con las siguientes dimensiones: SV, CP, PV, A, DE, TF y la Percepción Subjetiva de Ingresos (PS). Los modelos 2, 3, 4 y 5 son idénticos a los evaluados en el estudio 1.

Tabla 4.

Indicadores de bondad de ajuste de los modelos de $\mathrm{CV}$ analizados en el estudio 2

\begin{tabular}{lcccc}
\hline Modelo & Chi cuadrado & AIC & RMSEA & CFI \\
\hline Modelo 1 & 75.458 & 117.458 & .094 & .924 \\
Modelo 2 & 45.902 & 87.902 & .068 & .960 \\
Modelo 3 & 35.178 & 77.178 & .055 & .970 \\
Modelo 4 & 55.390 & 103.390 & .06 & .956 \\
Modelo 5 & $\mathbf{2 6 . 0 8 0}$ & $\mathbf{6 2 . 0 8 0}$ & $\mathbf{. 0 6 2}$ & $\mathbf{. 9 7 6}$ \\
\hline
\end{tabular}

Como se puede observar en la tabla 4, el modelo 5 (véase figura 1) es el que presenta los mejores indicadores de ajuste, analizando en su conjunto tanto índices absolutos como relativos (Hu \& Bentler, 1999).

\section{Análisis de confiabilidad}

Al igual que en el estudio se realizó un análisis de consistencia interna del instrumento que refleja el modelo de mejor ajuste (véase tabla 5). Los resultados son conceptualmente similares a los del estudio 1. 
Tabla 5.

Consistencia interna de la Escala de CV propuesta, Estudio 2

\begin{tabular}{clcccccc}
\hline \multirow{2}{*}{ Método } & \multicolumn{2}{c}{ Dimensión } & $\alpha$ & Dimensión & $\alpha$ & Método & $\alpha$ \\
\cline { 2 - 8 } Alfa de Cronbach & DE & .22 & $\mathrm{~A}$ & .77 & Correlación entre las partes & .79 \\
& $\mathrm{CP}$ & .77 & $\mathrm{SV}$ & .84 & & \\
& $\mathrm{PV}$ & .77 & $\mathrm{TF}$ & .76 & & \\
\end{tabular}

*Nota: $\alpha$ Valor coeficiente); DE (Dominio del entorno); CP (Crecimiento Personal); PV (Propósito en la vida); A (Auto aceptación); SV (Satisfacción con la vida); TF (Equilibrio Trabajo y Familia)

\section{DISCUSIÓN}

Los resultados de este segundo estudio muestran que el modelo de $\mathrm{CV}$ propuesto posee los mejores indicadores de ajuste a los datos, comparado con otros modelos alternativos. Se observa, nuevamente, que la dimensión objetiva de la CV queda fuera de la estructura factorial de dicho modelo. Tampoco parece ser relevante para el modelo formulado la percepción que las personas poseen de su situación financiera o económica. Aun cuando es un componente meramente subjetivo, el objeto al que refiere parece estar fuera de lo que las personas entienden en general por CV.

Por último, cabe destacar que la estabilidad del modelo persiste aun cuando las personas que responden pueden ser o no, trabajadoras. Del mismo modo, el lugar geográfico de la muestra no parece ser una explicación adecuada para entender la estructura del modelo, dado que las personas que responden se encuentran a una gran distancia una de otra, las condiciones geográficas son diferentes, y la estructura, no obstante, se mantiene.

\section{DISCUSIÓN GENERAL}

Los resultados de los dos estudios presentados muestran que existen elementos comunes entre diferentes constructos relacionados previamente en la literatura sobre CV. Este factor común obtenido se ha denominado Modelo de Calidad de Vida, y es el resultado de la reducción de diversos ítems que evalúan este constructo en diversos ámbitos disciplinarios. Las dimensiones de esta estructura dan mejor cuenta de los datos recogidos que otros modelos alternativos evaluados y que contienen indicadores tanto objetivos como subjetivos de la CV.

El modelo de CV obtenido posee similitudes conceptuales al planteado por Keyes, Shmotkin \& Ryff(2002), en donde el Bienestar está compuesto por aspectos relativos tanto al Bienestar Subjetivo como Psicológico. En dicha investi- gación se reconoce que, aunque ambos enfoques evalúan el bienestar, son conceptos diferenciados que apuntan a distintas características y concepciones del mismo, pero que están relacionados en algunas de sus dimensiones. En el presente trabajo se considera, no obstante, que el constructo obtenido implica aspectos más amplios que aquellos relativos a los ámbitos hedónicos y eudaimónicos. Además, que no todas las dimensiones del Bienestar son relevantes para él. Si bien es cierto que esto puede deberse al exigente criterio de selección utilizado, lo cierto es que de esa manera se obtiene una adecuada variabilidad con solo unos pocos ítems, lo que puede ayudar también a la intervención.

En línea con lo anterior, el modelo formulado agrupa aspectos asociados al Equilibrio TF, algo que no ocurre en los modelos más clásicos. Específicamente, la interacción positiva entre TF, en donde el bienestar familiar experimentado por las personas influiría positivamente y fomentaría su satisfacción con el trabajo. Estos resultados coinciden con trabajos previos que muestran la relevancia que para las personas tienen los aspectos familiares a la hora de evaluar su satisfacción vital o felicidad, independiente de su lugar de origen, edad o sexo (Diener, Suh, Lucas \& Smith, 1999; Edwards \& Rothbard, 2000; Moyano \& Ramos, 2007). Del mismo modo, coinciden con la idea de que la interacción de la vida laboral y familiar se influyen recíprocamente (Casas, Repullo, Lorenzo \& Cañas, 2002) y que las emociones y conductas de una esfera (familiar o laboral), son transferidas a la otra, de modo que si una persona ha tenido un buen día en su empleo, transferirá su bienestar al hogar, y viceversa (Moyano \& Jiménez, 2010).

Por otro lado, si bien existe un amplio marco conceptual acerca de la relación entre el crecimiento económico y la CV (p.ej., Easterlin \& Angelescu, 2012), los resultados obtenidos en este estudio no han dado cuenta de una mejor medida de la CV considerando la situación económica de quienes han respondido (medida a través de los indicadores 
habitualmente utilizados para reconocer esta variable de manera objetiva), ni tampoco a través de la percepción que la persona posee de dicha situación (Wolbring et al., 2011).

Esto sugiere fuertemente que el ámbito económico no es relevante para determinar la $\mathrm{CV}$, lo que puede observarse también en los datos poblacionales chilenos. Según cifras del Banco Mundial, el ingreso per cápita promedio del país se encuentra por encima de los 15 mil dólares anuales http:// datos.bancomundial.org/pais/chile). Este dato es relevante ya que, de acuerdo con diferentes estudios, el ingreso económico y el bienestar tienden a relacionarse cuando aquél es menor a 15 mil dólares. Sin embargo, el bienestar de una persona tiende a no relacionarse con su ingreso económico cuando este es superior a dicha cifra (Diener \& Seligman, 2004; Easterling, 1992; Oishi, Kesebir \& Diener, 2011; Smith, Diener, \& Wedell, 1989,). Existen investigaciones que dicen, incluso, que el aumento del crecimiento económico de un país genera un retroceso en el bienestar poblacional, ya que se relaciona fuertemente con un aumento en la tasa de suicidios (Moyano \& Barría, 2006).

Este trabajo no está exento de limitaciones. En primer lugar, en el estudio 2, tanto la dimensión de percepción subjetiva de ingresos, como la dimensión de autonomía están compuestas por pocos reactivos, lo que reduce la varianza explicada para una potencial dimensión al existir poca variabilidad en las respuestas (véase Batista \& Coenders, 2000). Futuros estudios pueden mejorar esta limitación incluyendo un número mayor de ítems por dimensión. No obstante, se considera que la inclusión de nuevas variables no debería generar diferencias sustantivas con los indicadores generales ya observados. Una de las principales razones es que cada ítem escogido posee un peso factorial alto en la determinación de su dimensión. Por ello, un aumento en el número de reactivos debería complementar la varianza explicada total actual. Sin embargo, es un aspecto a explorar en el futuro.

Otra limitación tiene que ver con algunos indicadores de consistencia interna cuyos valores se encuentran bajo el valor mínimo aceptable. Es el caso de la dimensión dominio del entorno, que posee una confiabilidad baja o deficiente en ambos estudios. Gallardo \& Moyano (2012), proponen que se pueden colapsar los ítems de Dominio del Entorno y Propósito en la Vida, creando un nuevo factor con mejores indicadores de fiabilidad. Utilizando los datos recogidos para los estudios descritos, se realizó el análisis propuesto, encontrándose una mejora de la confiabilidad para la variable combinada. No obstante, al realizar el análisis factorial confirmatorio con un modelo de cinco dimensiones (en donde se colapsan el dominio y el propósito), se observa que los indicadores de ajuste no mejoran significativamente y que incluso, algunos de ellos empeoran (véase tabla 3).
Futuros trabajos deberán abordar esta problemática, incorporando nuevos ítems de medida. Finalmente, si bien las variables (y escalas) consideradas en el diseño del Estudio 1 fueron seleccionadas sobre la base de investigaciones que las han relacionado sistemáticamente con la CV (Land, Michalos \& Sirgy, 2012), otras no lo fueron. Por ejemplo, una medida de Bienestar Social complementa la visión tradicional del bienestar con el ámbito social del ser humano (Blanco \& Díaz, 2007). Del mismo modo, incorporar una medida de CV objetiva adicional a la utilizada en estos estudios (p.ej., CV urbana, Orellana et al., 2011), podría informar de aspectos complementarios no registrados en la medida objetiva utilizada en los estudios del presente trabajo.

Consideramos que los resultados de la presente investigación permiten analizar la pertinencia, no solo de las medidas de calidad de vida en diferentes disciplinas académicas y profesionales, sino también del constructo que las fundamenta. Los datos obtenidos sugieren que una mirada de la CV basada en los aspectos subjetivos parece dar mejor cuenta de los datos recogidos que una combinación objetivo - subjetivo. Esto abre discusiones, no solo con respecto a los procedimientos de análisis (como los ya revisados) sino, de manera más trascendental, a discusiones teóricas importantes (p.ej., Kahneman, Krueger, Schkade, Scharz \& Stone, 2006).

Por ejemplo, se podría entender que el concepto de calidad de vida, en sus componentes subjetivo y objetivo, es perjudicial para el avance conceptual y aplicado del mismo. En este trabajo, la consideración de estos aspectos no explica de manera adecuada los datos recogidos, lo que sugiere que sería tal vez más útil llamar "Bienestar" a los aspectos subjetivos de la calidad de vida (de acuerdo con la tradición psicológica), y a los aspectos objetivos, "Condición de Vida" (de acuerdo con la tradición económica). Esto llevaría a una mayor claridad en la definición de lo que se pretende estudiar (lo objetivo o subjetivo, por separado) y, como consecuencia, las estrategias utilizadas para la intervención social.

\section{REFERENCIAS}

Ardila, R. (2003). Calidad de vida: Una definición integradora. Revista latinoamericana de psicología, 35, 161-164.

Batista, J. \& Coenders, G. (2000). Modelos de ecuaciones estructurales. Madrid: La muralla.

Batista-Foguet, J., Coenders, G. \& Alonso, J. (2004). Análisis factorial confirmatorio: Su utilidad en la validación de cuestionarios relacionados con la salud. Medicina clínica, 122, 21-27. 
Blanco, A. \& Díaz, D. (2007). El bienestar social: Su concepto y medición. Psicothema, 17, 582-589.

Bradburn, N. (1969). The Structure of Psychological WellBeing. Alpine: Chicago.

Carrasco, M., Delgado, B., Barbero, M., Holgado, F. \& del Barrio, V. (2011). Propiedades psicométricas del Interpersonal Reactivity Index (IRI) en población infantil y adolescente española. Psicothema, 23, 824-831.

Casas, J., Repullo, J., Lorenzo, S. \& Cañas, J. (2002). Dimensiones y medición de la calidad de vida laboral en profesionales sanitarios. Revista de administración sanitaria, 6 , 143-160.

Gobierno de Chile (2009). Encuesta de Caracterización Socioeconómica Nacional. Ministerio de Planificación. Recuperado de http://www.ministeriodesarrollosocial.gob.cl/ casen2009/RESULTADOS_CASEN_2009.pdf.

Concha, D., Bilbao, M., Gallardo, I., Páez, D. \& Fresno, A. (2012). Sesgos cognitivos y su relación con el bienestar subjetivo. Salud y sociedad, 3, 115-129.

Cudeck, R. \& Brown, M. (1983). Cross-validation in covariance structures. Multivariate Research, 18, 147-167.

Cummins, R. \& Cahil, J. (2000). Avances en la comprensión de la calidad de vida subjetiva. Intervención psicosocial, 9, 185-198.

Díaz, D., Rodríguez-Carvajal, R., Blanco, A., Moreno-Jiménez, B., Gallardo, I., Valle, C. \& Van Dierendonck, D. (2006). Validación española de las escalas de bienestar psicológico de Ryff. Psicothema, 18, 572-577.

Diener, E. (1984). Subjetive Well-Being. Psychological Bulletin, 95, 542-575.

Diener, E. (1994). Assessing subjective well-being: Progress and opportunities. Social Indicators Research, 31, $103-157$.

Diener, E., Emmons, R., Larsen, A. \& Griffin, R. (1985). The satisfaction with life scale. Journal of Personality Assessment, 49, 71-75.

Diener, E. \& Seligman, M. (2004). Beyond money. Toward an economy of well-being. Psychological Science in the Public Interest, 5, 1-31.

Diener, E., Suh, E., Lucas, R. \& Smith, H. (1999). Subjective well-being: Three decades of progress. Psychological bulletin, 125, 276-302.

Easterling, R. (1992). Will raising the incomes of all increase the happiness of all? Journal of Economic Behavior and Organization, 27, 35-47

Edwards, J. \& Rothbard, N. (2000). Mechanism linking work and family: Clarifying the relationship between work and family constructs". Academy of Management, 25, 178-199.

Felce, D. \& Perry, J. (1995). Quality of life: Its definition and measurement. Research in Developmental Disabilities, 16, 51-74.

Gallardo, I. \& Moyano, E. (2012). Análisis psicométrico de las escalas Ryff (versión española) en una muestra de adolescentes chilenos. Universitas psychologica, 11, 931-939.
Greenwald, A. \& Pratkanis, A. (1988). On the use of "theory" and the usefulness of theory. Psychological Review, 95, 575-579.

Greenwald, A., Pratkanis, A., Leippe, R. \& Baumgardner, M. (1986). Under what conditions does theory obstruct research progress? Psychological Review, 93, 216-229

Hu, L. \& Bentler, P. (1999). Cutoff criteria for fit indexes in covariance structure analysis: Conventional criteria versus new alternatives. Structural Equation Modeling, 6, 1-55.

Juárez, F. \& Contreras, F. (2012). Calidad de vida y liderazgo. Influencia de la calidad de vida percibida del directivo colombiano sobre sus prácticas de liderazgo. Acta Colombiana de Psicología, 15, 119-130.

Kahneman, D., Krueger, A., Schkade, D., Scharz, N. \& Stone, A. (2006). Would you be happier if you were richer? A focusing illusion. Science, 312, 1908-1910.

Land, K., Michalos, A. \& Sirgy, J. (2012). Prologue: The development and evolution of research on social indicators and quality of life (QOL). En K., Land, A., Michalos y J., Sirgy (Eds.), Handbook of social indicators and quality of life research (pp. 113 - 136). New York: Springer.

Lee, D. \& Sirgy, J. (2012). Consumer well-Being (CWB): Various conceptualizations and measures. En K., Land, A., Michalos y J., Sirgy (Eds.), Handbook of social indicators and quality of life research (pp. 113 - 136). New York: Springer.

Leiva, M. \& Gallardo, I. (2013). Validation of the short posttraumatic stress disorder rating interview (SPRINT-E) in a sample of people affected by F-27 Chilean earthquake and tsunami. Anales de psicología, 29, 328-334.

Malley, J., Towers, A., Netten, A., Brazier, J., Forder, J., \& Flynn, T. (2012). An assessment of the construct validity of the ASCOT measure of social care-related quality of life with older people. Health Quality of Life Outcomes, 10, 1477-7525.

Midlife in the United States (MIDUS). (2015). A national longitudinal study of health and well-being. Recuperado de http://midus.wisc.edu/.

Montazeri, A., Harirchi, A., Shariati, M., Garmaroudi, G., Ebadi, M., \& Fateh, A. (2003). The 12-item General Health Questionnaire (GHQ-12): translation and validation study of the Iranian version. Health and Quality of Life Outcomes, 1, 66.

Moyano, E. (Ed.). (2010). Calidad de vida y psicología en el bicentenario de Chile. Santiago de Chile: Mármor.

Moyano, E. \& Barría, R. (2006). Suicidio y producto interno bruto (PIB) en Chile: Hacia un modelo predictivo. Revista latinoamericana de psicología, 38, 343-359.

Moyano, M. \& Jiménez, A. (2010). Factores laborales de equilibrio entre trabajo y familia: Medios para mejorar la calidad de vida. En E., Moyano (Ed.). Calidad de vida y psicología en el bicentenario de Chile (pp. 249-272). Santiago de Chile: Mármor.

Moyano, E. \& Ramos, N. (2007). Bienestar subjetivo: Midiendo satisfacción vital, felicidad y salud en población chilena de la región del Maule. Universum, 2, 177-193. 
Oishi, S., Kesebir, S. \& Diener, E. (2011). Income inequality and happiness. Psychological Science, 22, 1095-1100.

Orellana, A., Bannen, P., Fuentes, L., Gilabert, H. \& Pape, K. (2011). Informe final indicador calidad de vida urbana (ICVU). Recuperado de http://www.estudiosurbanos.uc.cl/ wp-content/uploads/2011/05/Informe-Final-ICVU1.pdf

Organisation for Economic Co-operation and Development (OECD). (2015). Better life index [online]. Recuperado de http://www.oecdbetterlifeindex.org/es/

Oviedo, H. \& Campo - Arias, A. (2005). Aproximación al uso del coeficiente alfa de cronbach. Revista colombiana de psiquiatría, 34, 572-580.

Petrocelli, J. (2003). Factor validation of the consideration of future consequences scale: Evidence for a short version. The Journal of Social Psychology, 143, 405-413.

Riquelme, E., Rojas, A. \& Jiménez, A. (2012). Perspectivas analíticas sobre la dinámica social. Equilibrio trabajo, familia, apoyo familiar, autoeficacia parental y funcionamiento familiar percibidos por funcionarios públicos de Chile. Trabajo y sociedad, 18, 203-215.

Rocha, K., Pérez, K., Rodríguez-Sanz, M., Borrell, C. \& Obiols, J. (2011). Propiedades psicométricas y valores normativos del General Health Questionnaire (GHQ-12) en población general española. International Journal of Clinical and Health Psychology, 11, 125-139.

Ruiz, M., Pardo, A. \& San Martín, R. (2010). Modelos de ecuaciones estructurales. Papeles del psicólogo, 31, 34-45.

Ryan, R. \& Deci, E. (2001). On happiness and human potentials: A review of research on hedonic and eudaimonic wellbeing. Annual Reviews, 52, 141-166.

Ryff, C. (1989). Happiness is everything or is it? Explorations on the meaning of psychological well-being. Journal of Personality and Social Psychology, 57, 1069-1081.

Ryff, C. \& Keyes, C. (1995). The structure of psychological well-being revisited. Journal of Personality and Social Psychology, 69, 719-727.
Ryff, C. \& Singer, B. (1998). Middle age and well-being. En H., Friedman (Ed.), Encyclopedia of mental health (pp. 707719). San Diego, CA: Academic Press.

Sirgy, M., Michallos, A., Ferriss, A., Easterlin, R., Patrick, D. \& Pavot, W. (2006). The quality-of-life (QOL) research movement: Past, present and future. Social indicators research, 76, 343- 466.

Schreiber, J., Stage, F., King, J., Nora, A. \& Barlow, E. (2006). Reporting structural equation modeling and confirmatory factor analysis results: A review. The Journal of Educational Research, 99, 323-337.

Smith, H., Dienner, E. \& Wedell, H. (1989). Intrapersonal and social comparison determinants of happiness: A range-frecuency analysis. Journal of Personality and Social Psychology, 56, 317-325.

Springer, K. \& Hauser, R. (2006). An assessment of the construct validity of Ryff's scales of psychological well-being: Method, mode, and measurement effects. Social Science Research, 35, 1080-1102.

Springer, K., Hauser, R. \& Freese, J. (2006). Bad news indeed for Ryff's six factor model of well - being. Social Science Research, 35, 1120-1131.

Strathman, A., Gleicher, F., Boninger, D. \& Edwards, C. (1994). The consideration of future consequences: Weighing immediate and distant outcomes of behavior. Journal of Personality and Social Psychology, 66, 742-752.

Urzúa, A. \& Caqueo-Urízar, A. (2012). Calidad de vida: Una revisión teórica del concepto. Terapia psicológica, 30, 61-71.

Verdugo, A., Gómez, L. \& Arias, B. (2007). La escala integral de calidad de vida. Desarrollo y estudio preliminar de sus propiedades psicométricas. Siglo cero, 38, 37-56.

Wolbring, T. L, Keuschningg, M. \& Negele, E. (2011). Needs, comparisons, and adaptation: The importance of relative income for life satisfaction. European Sociological Review, 29(1), 86-104. 